\title{
Cancer Patterns Among Koreans in Seoul, Korea, and in Los Angeles, USA
}

\author{
Yoon-Ok Ahn
}

Although most are first-generation immigrants, the Korean population of Los Angeles, USA, showed a different pattern of cancer incidence to Korean residents of Seoul. Among Koreans in Los Angeles, the incidence of cancer, at all sites, was lower than in Seoul, both in males and females; among males aged 40-59 and females aged 30-49, the incidence was markedly lower. For several sites of cancer, for example the stomach, liver, and bladder in males and the cervix uteri and liver in females, the incidence for U.S. Koreans was significantly lower. Furthermore, for several sites, mean age at diagnosis was lower in Seoul than in Los Angeles.

For Korean males in Seoul, the seven leading sites of cancer, namely the stomach(26.4\%), lung(16.2\%), liver(14.4\%), colo-rectum(8.9\%), bladder $(3.9 \%)$, gallbladder $(3.8 \%)$, and pancreas $(2.7 \%)$, accounted for three-quarter(76.3\%) of all cancers occurring. For Korean males in Los Angeles, the seven leading sites were the stomach(22.9\%), lung(21.1\%), liver(11.1\%), colo-rectum(6.8\%), prostate(4.9\%), non-Hodgkin's lymphoma(3.3\%), and gallbladder(2.8\%), and comprised $72.9 \%$ of all cancers.

In females in Seoul, the seven leading cancer sites, comprising $75.8 \%$ of all cancers, were the cervix uteri(20.5\%), stomach(16.9\%), breast(11.6\%), colo-rectum(9.0\%), liver(6.7\%), lung(6.6 $\%)$, and thyroid(4.5\%). Among females in Los Angeles, the sites were the stomach(18.0\%), cervix uteri $(13.6 \%)$, breast $(13.3 \%)$, lung $(9.7 \%)$, colo-rectum $(9.6 \%)$, thyroid $(5.6 \%)$, and liver $(3.1 \%)$, and comprised $72.9 \%$ of all cancers. J Epidemiol, $1996 ; 6:$ S161-S164.

cancer, incidence, migrants, Korean, descriptive epidemiology

It is well known that cancer patterns among migrants have changed and are different to those among inhabitants of the homeland. The migrant population is therefore a valuable source for epidemiological studies, especially of etiology (1). The Korean population in Los Angeles Country, USA, migrated there very recently. Statistics from a sample survey(Korean Health Education, Information \& Referral Center in Los Angeles, Personal Communication) compiled in 1989 indicate that the majority of the Korean immigrant population there $(83.4 \%$ ) were born in Korea; i.e. they are the first generation of migrants. Only $3.6 \%$ have lived in the USA for 20 years or more, and $61.3 \%$ for nine years or less.

Though Korean migrants living in Los Angeles are a young migrant population, they showed a different pattern of cancer incidence to Koreans living in Seoul, Korea.

\section{SOURCE OF DATA}

Two sources of data were used to compare cancer patterns between the two populations: cancer incidence statistics for Seoul, 1991(2), and the cancer incidence data for Los Angeles County 1983-1987(3).

According to the 1990 population and housing census report in Korea, the total population of Seoul in that year was $10,603,247$. Through active and passive surveillance of the Seoul Cancer Registry, a total of 13,466 cancer cases $(6,965$ males, 6.501 fermales), newly diagnosed between July, 1991 and June, 1992, were identified and/or abstracted. The 1991 Seoul cancer incidence data were reported by Drs. Kim JP, Ahn YO, et al.

Cancer incidence data, 1983-1987, relating to Korean Americans in Los Angeles County were included in the $6^{\text {th }}$

Department of Preventive Medicine, Seoul National University College of Medicine, Seoul, Korea.

Address for correspondence : Yoon-OK Ahn, Department of Preventive Medicine, Seoul National University College of Medicine, 28 Yongon-dong Choungno-gu Seoul, 110-799, Korea. 
issue of 'Cancer Incidence in Five Continents', 1992. The source population was estimated to be 116,696 , as of 1985 , and a total of 620 cancer cases ( 310 males and 310 females) were identified between 1983-1987. It is said that over 300,000 Koreans are living in Los Angeles and its vicinity, but many of these have retained their Korean nationality.

\section{CANCER INCIDENCE PATTERNS BETWEEN THE TWO POPULATIONS, AT ALL SITES}

The incidence level of cancer, at all sites, among Korean Americans in Los Angeles(KA) was significantly lower than in Seoul(KS). The standardized KA incidence ratio was estimated to be 0.736 for males and 0.767 for females(Table 1). Especially among KA males aged 40-59 and KA females 3049, cancer incidence was markedly lower than that of KS(Fig. 1). Among KA, the mean age at first diagnosis was 63.5 and 62.0 years in males and females, respectively. The indirectly standardized mean age at first diagnosis among KS cancer cases (applying age-specific cancer incidence rates of $\mathrm{KS}$ to the KA population) was 57.4 years for males and 52.0 years for females. Among Koreans in Seoul, cancerous diseases in general occur at an earlier age and are more frequent than among KA.

The seven leading cancer sites and their corresponding percentages among KS males were the $\operatorname{stomach}(26.4 \%)$, lung $(16.2 \%)$, Liver(14.4\%), colo-rectum( $8.9 \%)$, bladder(3.9\%), gallbladder(3.8\%), and pancreas $(2.7 \%)$. These comprised three-quarter( $76.3 \%$ ) of all cancers. In Los Angeles, the leading sites were the $\operatorname{stomach}(22.9 \%)$, lung $(21.1 \%)$, liver(11.1\%), colo-rectum(6.8\%), prostate( 4.9 $\%)$, non-Hodgkin's lymphoma(3.3\%), and gallbladder $(2.8 \%)$, and they comprised $72.9 \%$ of all cancers. In females in Seoul, the seven leading cancer sites were the cervix uteri $(20.5 \%)$, stomach $(16.9 \%)$, breast(11.6\%), colo-rec- $\operatorname{tum}(9.0 \%)$, liver(6.7\%), lung(6.6\%), and thyroid(4.5\%); these comprised $75.8 \%$ of all cancers. For KA females, the sites were the stomach(18.0\%), cervix uteri(13.6\%), breast $(13.3 \%)$, lung $(9.7 \%)$, colo-rectum( $9.6 \%)$, thyroid(5.6\%), and liver(3.1\%). These accounted for $72.9 \%$ of all cancers.

\section{STOMACH CANCER}

The incidence level of stomach cancer among KS males was much higher than among $\mathrm{KA}$, for whom the standardized incidence ratio of this cancer was estimated to be 0.64 . For females there was no difference in incidence level between the two populations. For KS and KA, mean age at first diagnosis of stomach cancer was similar in males, but in females was 5-6 years earlier in KS(Table 2).

\section{LIVER CANCER}

In both males and females, the incidence level of liver cancer among KS was much higher than among KA, for whom the SIR of this cancer was estimated to be 0.60 and 0.45 in males and females, respectively. At first diagnosis of liver cancer, the mean age of KS males was 1.5 years less, and of KS females, 5 years less than of KA males and females, respectively (Table 2).

\section{LUNG CANCER}

In the KS population, mean age at first diagnosis was slightly less, but in this and in incidence level, there were no marked differences between the two populations(Table 2).

Table 1. A comparison of cancer incidence at all sites among Koreans in Seoul(KS) and in Los Angeles(KA).

\begin{tabular}{|c|c|c|c|c|c|c|}
\hline \multirow{2}{*}{ Indicator } & \multicolumn{3}{|c|}{ Male } & \multicolumn{3}{|c|}{ Female } \\
\hline & $\mathrm{KS}$ & KA & ratio & KS & $\mathrm{KA}$ & ratio \\
\hline ASR, World & 232.4 & 181.2 & 0.78 & 147.9 & 127.5 & 0.86 \\
\hline $\mathrm{CR}, 0-64$ & 13.0 & 9.0 & 0.69 & 10.3 & 7.6 & 0.74 \\
\hline CR, 0-74 & 28.4 & 17.9 & 0.63 & 17.2 & 14.5 & 0.84 \\
\hline SIR, KA & \multicolumn{3}{|c|}{$310 / 421=0.74^{*}$} & \multicolumn{3}{|c|}{$310 / 404=0.77 *$} \\
\hline Mean age(years) & 57.4 & 63.5 & & 52.0 & 62.0 & \\
\hline
\end{tabular}

ASR ; Age standardized incidence rate per 100,000 for world population

$\mathrm{CR} ;$ Cumulative incidence rate $(\%)$, for the age spans $0-64$ or $0-74$

SIR ; Standardized incidence ratio of KA using indirect $\operatorname{method}(* ; p<.05)$

Mean age; Average age at first diagnosis, those of $\mathrm{KA}$ are crude, but those of KS are indirectly standardized for comparison. 
Table 2. A comparison of the incidence of several leading cancer sites among Koreans in Seoul(KS) and in Los Angeles(KA).

\begin{tabular}{|c|c|c|c|c|c|c|}
\hline \multirow{2}{*}{ indicator } & \multicolumn{3}{|c|}{ Male } & \multicolumn{3}{|c|}{ Female } \\
\hline & $\mathrm{KS}$ & $\overline{K A}$ & ratio & KS & $\mathrm{KA}$ & ratio \\
\hline \multicolumn{7}{|l|}{ Stomach } \\
\hline ASR, World & 60.6 & 41.5 & 0.68 & 24.6 & 22.9 & 0.93 \\
\hline CR, 0-74 & 7.6 & 3.7 & 0.49 & 3.1 & 2.8 & 0.90 \\
\hline SIR, KA & \multicolumn{3}{|c|}{$70 / 110=0.64^{*}$} & \multicolumn{3}{|c|}{$52 / 65=0.80^{*}$} \\
\hline Mean age(years) & 58.2 & 59.1 & & 55.6 & 61.4 & \\
\hline \multicolumn{7}{|l|}{ Liver } \\
\hline ASR, World & 30.8 & 20.1 & 0.65 & 9.7 & 3.9 & 0.40 \\
\hline $\mathrm{CR}, 0-74$ & 4.0 & 1.9 & 0.48 & 1.3 & 0.4 & 0.31 \\
\hline SIR, KA & \multicolumn{3}{|c|}{$39 / 65=0.60^{*}$} & \multicolumn{3}{|c|}{$10 / 22=0.45^{*}$} \\
\hline Mean age(years) & 54.9 & 56.4 & & 59.8 & 64.8 & \\
\hline \multicolumn{7}{|l|}{ Lung } \\
\hline ASR, World & 37.3 & 38.3 & 1.03 & 9.7 & 12.4 & 1.28 \\
\hline CR, 0-74 & 4.8 & 4.5 & 0.94 & 1.2 & 1.4 & 1.17 \\
\hline SIR, KA & \multicolumn{3}{|c|}{$60 / 61=0.98$} & \multicolumn{3}{|c|}{$28 / 23=1.22$} \\
\hline Mean age(years) & 62.6 & 64.3 & & 61.7 & 64.0 & \\
\hline \multicolumn{7}{|l|}{ Colorectum } \\
\hline ASR, World & 20.4 & 12.3 & 0.60 & 13.2 & 12.3 & 0.93 \\
\hline $\mathrm{CR}, 0-74$ & 2.2 & 1.2 & 0.55 & 1.6 & 1.3 & 0.81 \\
\hline SIR, KA & \multicolumn{3}{|c|}{$19 / 38=0.50^{*}$} & \multicolumn{3}{|c|}{$29 / 32=0.91$} \\
\hline Mean age(years) & 57.5 & \multicolumn{2}{|c|}{67.6} & 58.4 & \multicolumn{2}{|l|}{63.5} \\
\hline Bladder & & & & Breast & & \\
\hline ASR, World & 8.8 & 4.7 & 0.53 & 17.0 & 16.9 & 1.00 \\
\hline $\mathrm{CR}, 0-74$ & 1.2 & 0.3 & 0.25 & 1.7 & 2.0 & 1.18 \\
\hline SIR, KA & \multicolumn{3}{|c|}{$7 / 15=0.47^{*}$} & \multicolumn{3}{|c|}{$48 / 52=0.93$} \\
\hline Mean age(years) & 61.5 & 70.9 & & 46.7 & 48.6 & \\
\hline \multicolumn{7}{|l|}{ Cervix uteri } \\
\hline ASR, World & & & & 22.9 & 17.4 & 0.76 \\
\hline CR, 0-74 & & & & 3.3 & 2.2 & 0.66 \\
\hline SIR, KA & & & & \multicolumn{3}{|c|}{$49 / 91=0.54^{*}$} \\
\hline Mean age, crude & & & & 47.7 & 53.8 & \\
\hline
\end{tabular}

ASR ; Age standardized incidence rate per 100,000 for world population

CR ; Cumulative incidence rate(\%), for the age spans 0-74

SIR ; Standardized incidence ratio of KA using indirect method( $* ; \mathrm{p}<.05)$

Mean age; Average age at first diagnosis, those of KA are crude, but those of KS are indirectly standardized for the comparison. 

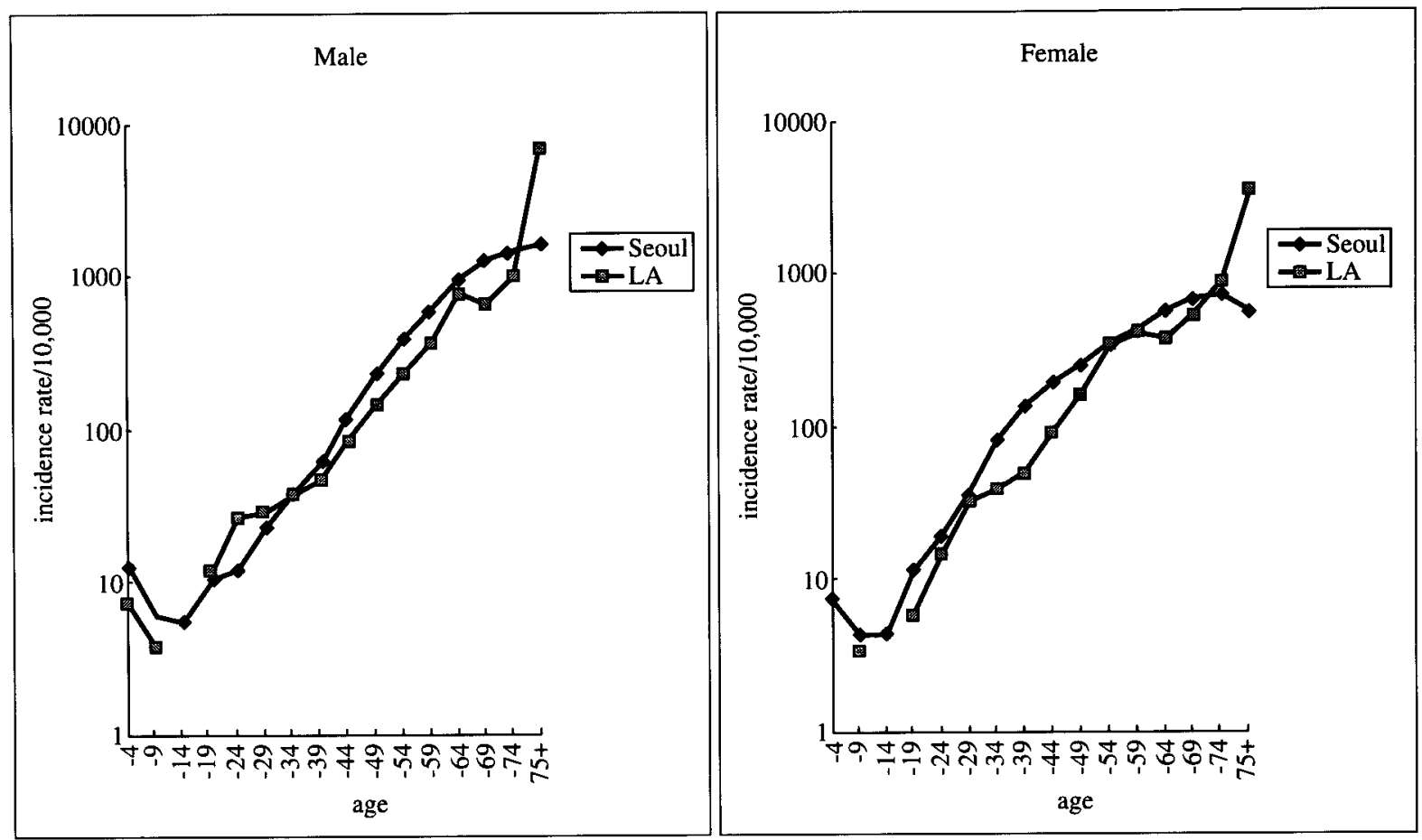

Figure 1. Age incidence curve of cancer at all sites, among Koreans in Seoul (1991) and in Los Angeles (1983-1987).

\section{COLO-RECTAL CANCER}

The incidence level of colo-rectal cancer among KS males was twice as high as among KA males(Tabel 2). For females, however, there was no difference in incidence levels between the two populations. Mean ages of KS and KA at first diagnosis of such cases were strikingly different; compared with KA, among KS males and females occurred ten and five years earlier, respectively.

\section{BLADDER CANCER IN MALES}

The incidence level of bladder cancer among KS males was more than twice as high as among KA males, and mean age at first diagnosis was nearly 9 years earlier in $\mathrm{KS}$ than in KA(Table 2).

\section{BREAST CANCER, FEMALES}

For breast cancer, there were no marked differences between the two populations in incidence levels and mean age at first diagnosis, though in the KS population, mean age was slightly less.

\section{CANCER OF UTERINE CERVIX}

The incidence level of cervical cancer in KS was much high than in KA. The SIR of this cancer amnog KA was estimated to be 0.54 (Table 2). Mean age at first diagnosis of cervical cancer cases in KS was 6 years earlier than in KA.

\section{REFERENCES}

1. Haenszel W. Migrant studies, In: Schottenfel D, Fraumeni J Jr. (eds.). Cancer Epidemiology and Prevention, Philadelphia, W B Saunders Company, 1982: 194-207.

2. Kim JP, Park IS, Ahn YO, et al. 1991 Cancer Incidence in Seoul, Korea : Results of the Implementation Study of the Seoul Cancer Registry, J Korean Med Science, 1995;10:74-84.

3. Parkin DM, Muir CS, Whelan SL, et. al .(eds.) Cancer Incidence in Five Continents, Vol. VI, IARC Scientific Publication No. 120, International Agency for Research on Cancer, Lyon, France, 1992: 351-353. 